\title{
BMJ Open Association between prescription of hypnotics/anxiolytics and mortality in multimorbid and non-multimorbid patients: a longitudinal cohort study in primary care
}

Kristjan Linnet (D) , ${ }^{1}$ Johann Agust Sigurdsson, ${ }^{1,2}$ Margret Olafia Tomasdottir, ${ }^{3}$ Emil Larus Sigurdsson, ${ }^{1,3}$ Larus Steinthor Gudmundsson ${ }^{4}$

To cite: Linnet $K$,

Sigurdsson JA, Tomasdottir M0, et al. Association between prescription of hypnotics/ anxiolytics and mortality in multimorbid and nonmultimorbid patients: a longitudinal cohort study in primary care. BMJ Open 2019;9:e033545. doi:10.1136/ bmjopen-2019-033545

- Prepublication history and additional material for this paper are available online. To view these files, please visit the journal online (http://dx.doi. org/10.1136/bmjopen-2019033545).

Received 09 August 2019 Revised 31 October 2019 Accepted 12 November 2019

Check for updates

(C) Author(s) (or their employer(s)) 2019. Re-use permitted under CC BY-NC. No commercial re-use. See rights and permissions. Published by BMJ.

For numbered affiliations see end of article.

Correspondence to

Kristjan Linnet;

kristjan.linnet@heilsugaeslan.is

\section{ABSTRACT}

Objectives To assess the risk of mortality in primary care patients, multimorbid ( $\geq 2$ chronic conditions) or not, prescribed hypnotics/anxiolytics.

Design A longitudinal cohort study

Setting Primary healthcare in the Reykjavik area.

Participants 114084 individuals (aged 10-79 years, average 38.5, SD 18.4) contacting general practitioners during 2009-2012 (mortality follow-up to 31 December 2016). Of those, the reference group comprised 58560 persons who were neither multimorbid nor had redeemed prescriptions for hypnotics/anxiolytics. Participants (16 108) redeeming prescriptions for hypnotics/anxiolytics on a regular basis for 3 consecutive years were considered as consistent, long-term users. They were subdivided into low-dose (1-300 defined daily doses (DDD)/3 years), medium-dose (301-1095 DDDs/3 years) and high-dose users (>1095 DDDs/3 years). All six groups taking these drugs were compared with the reference group.

Main outcome measures All-cause mortality.

Results HRs were calculated with the no multimorbidityno drug group as a reference, using Cox proportional hazards regression model adjusting for age, sex and the number of chronic conditions $(n=111767)$, patients with cancer excluded. During follow-up, 516358 person-years in total, 1926 persons died. Mean follow-up was 1685 days (4.6 years), range $1-1826$ days (5.0 years). For all multimorbid patients who took no drugs the HR was 1.14 (95\% Cl 1.00 to 1.30) compared with those without multimorbidity. HRs in the non-multimorbid participants varied from 1.49 to 3.35 (95\% Cl ranging from 1.03 to 4.11) with increasing doses of hypnotics/anxiolytics, and correspondingly from 1.55 to 3.52 (1.18 to 4.29) in multimorbid patients.

Conclusions Mortality increased in a dose-dependent manner among both multimorbid and non-multimorbid patients taking hypnotics/anxiolytics. This increase was clearly associated with prescribing of these drugs. Their use should be limited to the recommended period of 2-4 up to 6 weeks; long-term use may incur increased risk and should be re-examined.

\section{INTRODUCTION}

In the last decades, various studies have expressed concerns about possible links
Strengths and limitations of this study

- Our study is the first population-based longitudinal cohort study to compare dose-dependent mortality associated with long-term (3-year consistent) prescribing of hypnotics/anxiolytics in multimorbid versus non-multimorbid patients.

- We retrieved International Classification of Diseases and Related Health Problems 10th Version diagnoses from a large data set of medical records of healthcare centres serving two-thirds of the entire population and linked with data on redeemed prescriptions from the national drug prescription database and mortality data from the national mortality registration database.

- Information on some confounding factors was lacking and confounding by indication cannot be avoided, so even after adjusting for chronic conditions some residual confounding may still be present.

- The definition of multimorbidity is not universally standardised and may be heterogeneous to a certain extent.

between the use of hypnotic/anxiolytic benzodiazepine drugs and increased mortality. ${ }^{1-4}$ These studies have, however, shown inconsistent results. This can partly be explained by differences in methodology and design. Other studies have not lent support to a strong association of hypnotic use with increased mortality. ${ }^{5-7}$ A prospective study found that the risk of mortality was not significantly associated with hypnotic use regardless of type and duration. ${ }^{8}$ A retrospective cohort study correcting for a range of potential confounders found an association with significantly increased risk of mortality. ${ }^{9}$

Pharmacological properties of hypnotics could have an influence on mortality. Benzodiazepines and related drugs have respiratory suppressant effects which can be 
increased in combination with other respiratory depressant drugs or alcohol. ${ }^{10}$ Recently, the focus has been on opioids in the USA where a considerable number of opioid users were coadministered benzodiazepines leading to increased mortality. ${ }^{11}$ An Icelandic study found increased mortality in patients undergoing noncardiac surgery who received a preoperative prescription for both opioids and benzodiazepines. This was not the case when opioids or benzodiazepines were prescribed separately. ${ }^{12}$ The use of psychotropic drugs leads to risk of falls and fractures, ${ }^{13} 14$ and hip fractures markedly increase mortality in the elderly. ${ }^{15}$ An increased suicide risk has been reported in persons taking these drugs leading to increased mortality. ${ }^{316}$ Other untoward effects could also increase mortality such as an increased risk of road traffic accidents, ${ }^{17}$ dementia ${ }^{18}$ and Alzheimer's disease. ${ }^{19}{ }^{20}$ Other studies do not support a causal association between benzodiazepine use and dementia. ${ }^{21} \mathrm{An}$ association was found between exposure to benzodiazepines and increased mortality from community-acquired pneumonia. ${ }^{22}$ To summarise, all this could contribute to increased mortality.

We are only aware of one prior general practice study clearly showing a dose-dependent relationship between mortality and use of these drugs. ${ }^{9}$ We consider that defining long-term use as 3 consecutive years of consistent use with up to 5 years of follow-up (3 years exposure +5 years follow-up $=8$ years $)$ in both multimorbid and non-multimorbid patients will further evidence the relationship between use of these drugs and mortality. Multimorbidity is known to be associated with increased mortality. ${ }^{23} 24$

The aim of this study is to analyse a possible difference in risk of mortality (hazard) in persons either multimorbid or not, prescribed hypnotics/anxiolytics or not, by comparing the different groups to patients who neither were multimorbid nor had been prescribed hypnotics/ anxiolytics. Furthermore, to find whether there could be a dose-dependent risk of mortality in long-term users of hypnotics/anxiolytics.

\section{MATERIALS AND METHODS \\ Design and setting}

The Primary Healthcare of the Capital Area (PHCA) includes most of the primary healthcare centres (PHC) in the Reykjavik area with a population of just over 200 000 . The PHCs are staffed by general practitioners (GP), midwives, nurses and other personnel. Information on age and sex of all patients who had contacted the PHCA during a 4-year period from 1 January 2009 to 31 December 2012 was retrieved from the PHCA medical records database, as well as the diagnosis (International Classification of Diseases and Related Health Problems 10th Version; ICD-10) for chronic conditions as described in a previous paper $^{25}$ (see online supplementary table $1)$. The data in the PHCA medical records database are stored under a unique personal identifier (ID) allocated to every inhabitant.

\section{Exposure}

The extracted data were linked through the patients' IDs with data on redeemed hypnotic/anxiolytic prescriptions in the Icelandic Medicine Register (IMR). All redeemed prescriptions for hypnotics/anxiolytics with the Anatomical Therapeutic Chemical (ATC) codes N05BA, N05CD and N05CF were retrieved and collected in the study database. The use of hypnotics/anxiolytics was classified into two categories: Z-drugs and benzodiazepines. Individual drugs prescribed are listed in online supplementary table 2 . In this study we employ the term intermittent user for those who were prescribed hypnotics/ anxiolytics occasionally during the 4-year period for less than 3 consecutive years, whereas long-term users are those who used hypnotics/anxiolytics consistently during at least 3 consecutive years. Low-dose users were defined as those who used 1-300 defined daily doses (DDD)/3 years, medium-dose users as those who used 301-1095 DDDs / 3 years and high-dose users as those who used $>1095$ DDDs/3 years. The upper limit for the low dose is three prescriptions per year according to clinical guidelines (4-6 weeks) over 3 consecutive years. High-dose use is one dose or more per day, also over 3 consecutive years $(365 \times 3=1095)$.

\section{Covariates}

In this paper we apply the commonly used definition of multimorbidity as the presence of two or more chronic medical conditions in the same person, and we use previously described conditions as the frame of reference. ${ }^{25}$ In the Cox proportional regression analysis, adjustment was made for the number of chronic conditions. The groups were as follows: no multimorbidity group (zero or one chronic condition) and multimorbidity group (2-3, $4-5,6-7$ or $8-15$ chronic conditions). The list of chronic conditions used in this study coded by the ICD-10 is to be found in online supplementary table 1 . Insomnia is not among the listed conditions as we presumed it was an inclusive part of the decision to prescribe a hypnotic. Distribution of age and sex in the cohort is to be found in table 1 showing baseline characteristics.

\section{Outcome}

Information on mortality in the cohort was extracted by linking the IDs with relevant data in the National Cause of Death Registry. When all relevant data for the study database had been linked through the subjects' IDs it was encrypted so the personal identity of the patients would not be revealed during the processing of the data set which was collected in a study database.

\section{Subject groups and statistical analysis}

The mortality of primary care patients, multimorbid or not, who were consistent users of hypnotics/anxiolytics during a 3-year period in the time interval from 1 January 2009 to 31 December 2012 was determined and compared with 
Table 1 Baseline characteristics of participants in the longitudinal cohort study in primary care in Iceland

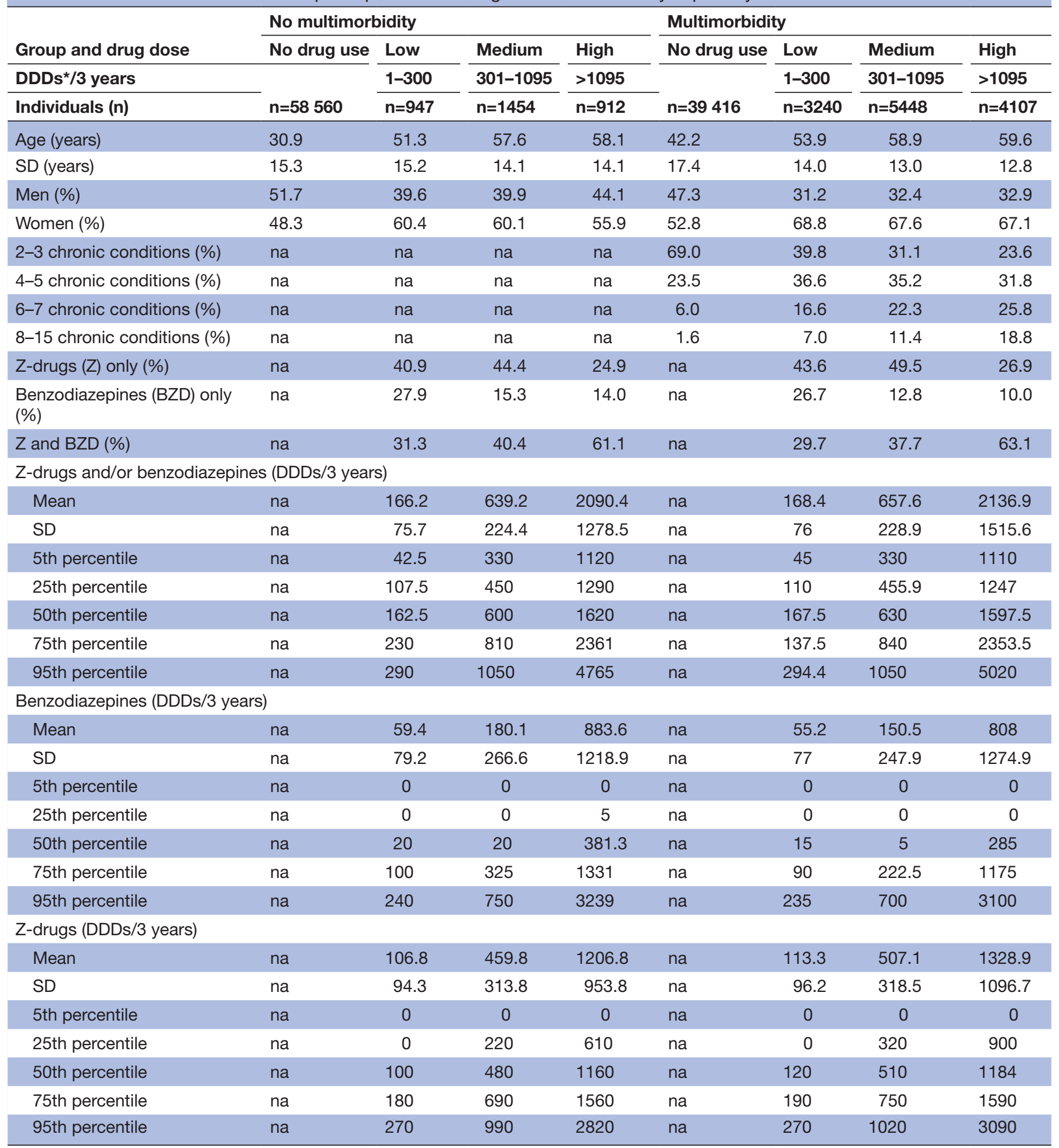

Multimorbidity: the presence of two or more chronic medical conditions in the same person.

${ }^{*}$ Defined daily doses of Z-drugs and/or benzodiazepines used during a 3-year recruitment period (from 2009 to 2012). na, not applicable.

non-multimorbid subjects not using hypnotics/anxiolytics (figure 1). A flow chart (figure 2) shows who were excluded in our study. We ended up with 114084 participants who could be divided into four groups: (A) 58560 persons who were neither multimorbid nor had been prescribed hypnotics/anxiolytics, (B) 12795 multimorbid patients who had been prescribed hypnotics/anxiolytics, (C) 39 416 multimorbid patients who had not been prescribed hypnotics/anxiolytics and, finally, (D) 3313 persons who were not multimorbid but had been prescribed hypnotics/ 


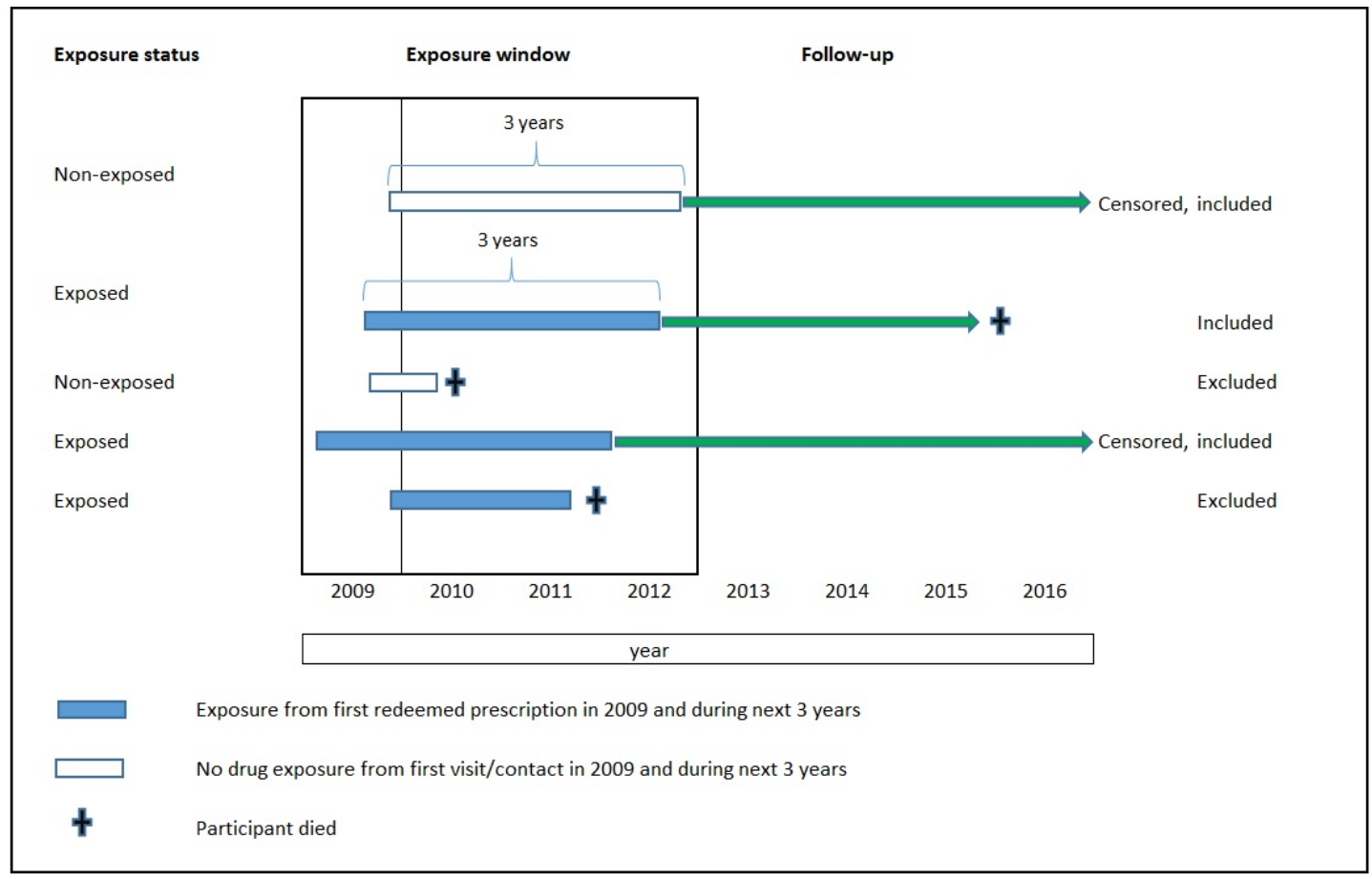

Figure 1 Participant inclusion/exclusion to the study. Schematic presentation of participant inclusion/exclusion to the study, their exposure to anxiolytics (ATC classification N05B) and/or hypnotics/sedatives (ATC classification N05C) during a 3-year recruitment period/exposure window. Participants contacted the Primary Healthcare of the Capital Area in Reykjavik and its adjacent towns during 2009, and the follow-up started 3 years after first prescription or first visit. Persons who redeemed prescriptions for the above-mentioned drugs for 3 consecutive years were included and considered consistent (long-term users). ATC, Anatomical Therapeutic Chemical.

anxiolytics. Thus, 16108 patients had redeemed prescriptions for hypnotics/anxiolytics in the relevant ATC classes on a regular basis for 3 consecutive years and were considered as consistent or long-term users. They were divided into multimorbid and non-multimorbid subjects. Both these groups were subdivided into low-dose users (1-300 DDDs/3 years), medium-dose users (301-1095 DDDs/3 years) and high-dose users ( $>1095$ DDDs/3 years), all six groups compared with those who neither were multimorbid nor took hypnotics/anxiolytics and were set as a reference. It can be argued whether patients who use 1-300 DDDs/ 3 years can be defined as consistent users. However, we include them in order to reveal if low-dose use during a long time is associated with mortality. For all the patients who did not die during follow-up, the follow-up ended 31 December 2016.

We used Cox proportional regression analysis to determine the relative risk, that is, HR for all-cause mortality during the follow-up while adjusting for age, sex and the number of chronic conditions (tables 2 and 3 ). The nonmultimorbid participants not using hypnotics/anxiolytics during the years 2009-2012 were used as a reference. For the Nelson-Aalen cumulative hazard we restricted the analysis to participants aged $40-79$ years in order to even the average age in the groups compared. Significance testing was two sided and based on a $5 \%$ probability level. We tested the Cox models for violations of the proportional hazard assumption. ${ }^{26} 27$ The assumption was not violated in the main analyses. Three sensitivity analyses were performed. In the first sensitivity analysis using subjects aged 10-69 years the proportional hazard assumption was violated. By left truncating the follow-up by 120 days the assumption was no longer violated. This resulted in somewhat lower HRs (by $4 \%-20 \%$, table 3, model 3 ). The second sensitivity analysis was performed including participants aged 10-59 years. No left truncation was needed in the regression analysis. The third sensitivity analysis included participants aged 10-79 years, but the maximum cumulative dose of hypnotics and/ or anxiolytics was 42 DDDs/year, thus approximating the maximum duration (6 weeks) of a clinically recommended drug treatment with hypnotic-sedatives. The software package used was Stata V.13 (StataCorp, College Station, TX, USA).

\section{Ethics approval}

The National Bioethics Committee and the Data Protection Authority in Iceland approved the study (VSN 16-151).

\section{Public and Patient Involvement}

No patients were involved in the research process at any stage. Patient consent not required.

\section{RESULTS}

At study entry the number of participants was 114084 ; 54400 men and 59684 women, with an average age of 
Contacted the Primary Health Care of the Capital Area in Iceland $(n=221,822)$

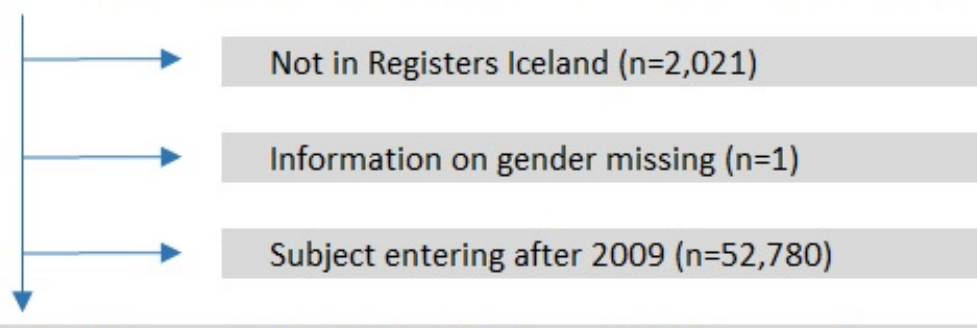

Subjects age 0 to 105 years ( $n=167,020 ; 78,317$ men; 88,703 women)

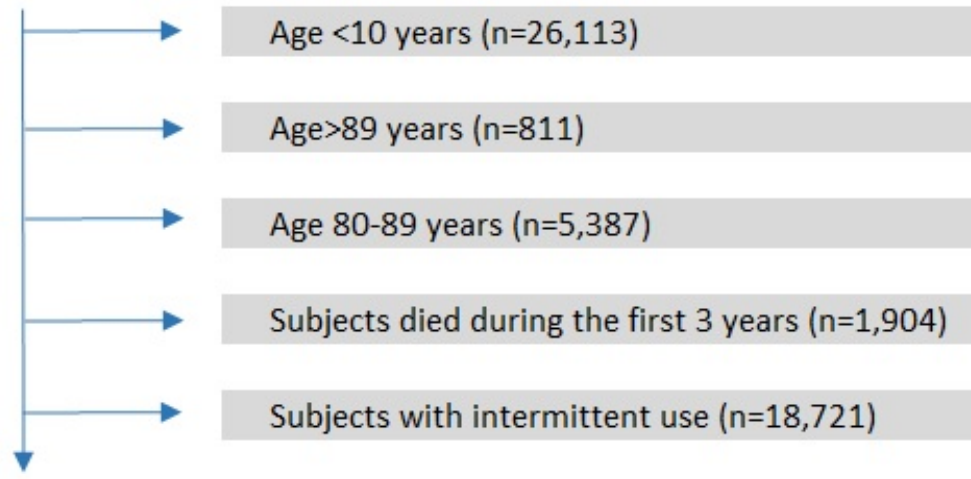

Study population age $10-79$ years ( $n=114,084 ; 54,400$ men; 59,684 women)

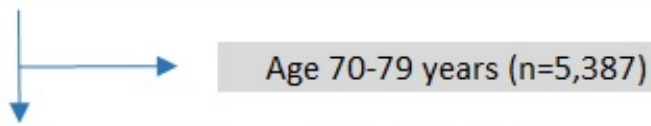

Sensitivity analysis population ( $n=107,121 ; 51,236$ men; 55,885 women)

Figure 2 Flow chart of participants included in the longitudinal cohort study in primary care in Iceland.

38.5 years, SD 18.4, and an age range of 10-79 years. Table 1 shows the baseline characteristics of the study participants. The average age of those who were neither multimorbid nor had used hypnotics/anxiolytics was 30.9 years. The multimorbid patients were 11 years older on the average. Individuals, either multimorbid or not, using hypnotics/anxiolytics were approximately 20-30 years older than those neither multimorbid nor using these drugs (table 1). Among multimorbid users of hypnotics/ anxiolytics the prevalence of those with six to seven or eight or more chronic conditions went hand in hand with increasing use (low, medium, high dose) of hypnotics/ anxiolytics (table 1). Compared with people without multimorbidity and no use of hypnotics/anxiolytics, those without multimorbidity but with a consistent 3-year hypnotic/anxiolytic use were at increased risk of mortality from all causes across all three dose categories, HRs varied from 1.49 to 3.35 depending on dose (results and $95 \%$ CIs in table 2, model 3). Likewise, compared with people without multimorbidity and no use of hypnotics/ anxiolytics, those with multimorbidity and with consistent 3-year hypnotic/anxiolytic use were at increased risk of mortality from all causes across the three dose categories,
HRs varying from 1.55 to 3.52 (table 2 , model 3 ). When excluding use of hypnotics/anxiolytics, multimorbid subjects compared with those without multimorbidity were at a borderline significantly increased risk of allcause mortality, the HR was 1.14 (95\% CI 1.00 to 1.30 , $\mathrm{p}=0.049$ ) (table 2, model 1). A similar pattern emerged when plotting the Nelson-Aalen cumulative hazard for the groups mentioned above, participants were at higher risk of mortality when using high doses of hypnotics/ anxiolytics (figure 3). Using the same reference group as above, a sensitivity analysis among participants 10-69 years old $(\mathrm{n}=107 \mathrm{121})$ revealed similar findings. Those who were not multimorbid but with a consistent 3-year use of hypnotics/anxiolytics were at increased risk of mortality from all causes across all three dose categories the HRs varying from 1.97 to 5.59 (results and 95\% CIs in table 3, model 3). Similarly, multimorbid participants using low, medium or high doses of hypnotics/anxiolytics were at increased risk of mortality, the HRs being from 2.37 to 5.93 (table 3, model 3).

In the second sensitivity analysis participants aged 10-59 years were included. The endpoints were too few, both in the low-dose and the medium-dose groups, for 


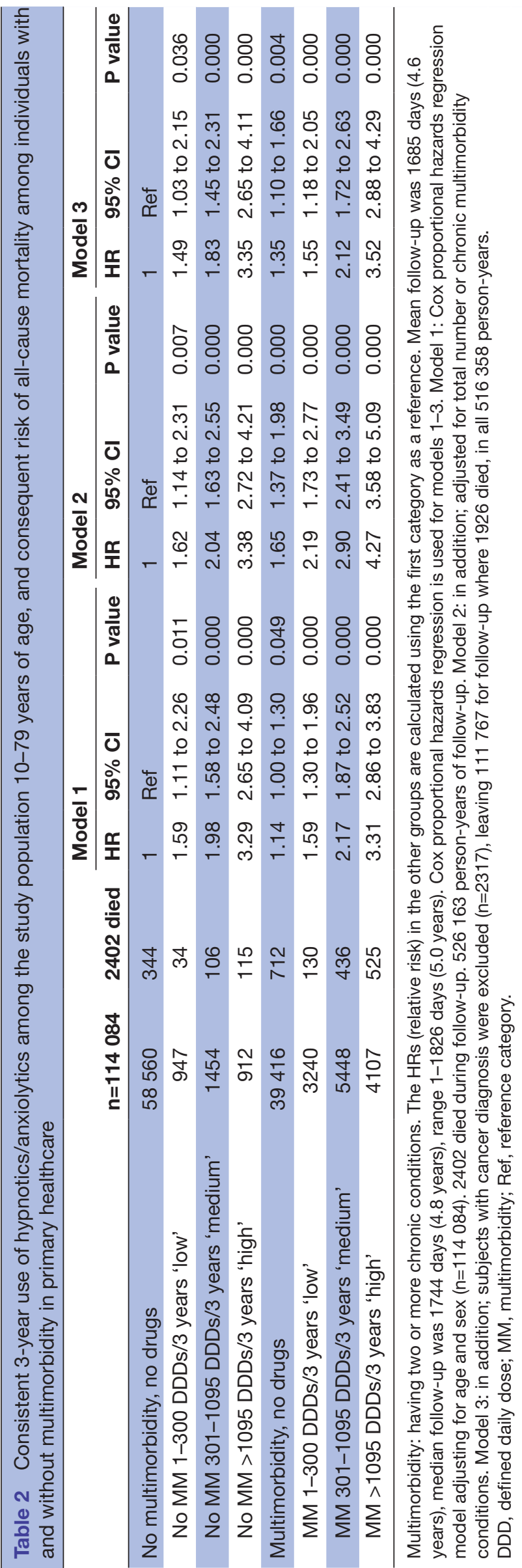

calculating HR. When omitting patients with cancer, the adjusted HR was 8.96 (95\% CI 5.72 to 14.02 , n=337, 13 deaths) for those without multimorbidity and high dose of hypnotics/anxiolytics. Similarly, for those with multimorbidity and high dose of hypnotics/anxiolytics, the adjusted HR was 11.92 (95\% CI 7.33 to 19.37 , n=1905, 102 deaths). The third sensitivity analysis where the annual maximum cumulative dose of hypnotics/anxiolytics was 42 DDDs (126 DDDs/3 years), the average annual dose of hypnotics/anxiolytics for the first year was 22.8 DDDs, $\mathrm{SD}=10.5 ; 20.0 \mathrm{DDDs}, \mathrm{SD}=10.8$ for the second year and 20.4 DDDs, $S D=10.9$ for the third year. Among those without multimorbidity $(n=115)$ there was only one death during the follow-up and therefore, an HR is not reported here. For those with multimorbidity HR was 1.13 (95\% CI 0.53 to $2.40, \mathrm{n}=693,12$ deaths).

Participants with two or three chronic conditions were not at increased risk of mortality compared with those with zero or one chronic condition. The risk of mortality when chronic conditions were four or five rose to HR 1.09 (95\% CI 0.97 to 1.29 ) for those aged $10-79$ years, and HR 1.21 (95\% CI 1.03 to 1.43 ) for ages $10-69$ years. The greatest risk of mortality was linked to having six to seven, and eight or more chronic conditions (online supplementary table 3 and 4 ).

\section{DISCUSSION}

\section{Principal findings}

Consistent long-term users of benzodiazepines/Z-drugs, either multimorbid or not, were subdivided into low-dose users (1-300 DDDs/3 years), medium-dose users (3011095 DDDs/ 3 years) and high-dose users (>1095 DDDs/3 years). The mortality among both the multimorbid and non-multimorbid patients prescribed these drugs over a period of 3 years increased in a dose-dependent manner. This increase was clearly associated with the prescribing of these drugs. In this paper we often apply the term use when strictly speaking it involves prescribing.

\section{Strengths}

This is a comprehensive study covering approximately two-thirds of the total population where all contacts with the vast majority of the healthcare centres in the area are registered in the medical records database. The distribution according to age and sex is the same as for the population at large so the cohort is truly representative. By linking the IDs to the data on redeemed prescriptions in the IMR during the study period we were able to cover all the prescriptions issued by the GPs.

\section{Limitations}

The definition of multimorbidity is not universally standardised. Although there seems to be a broad consensus on a considerable number of different chronic diseases covered by the definition of two or more chronic diseases in one person, it does not cover all diseases that have been included by various authors, thus making the definition 


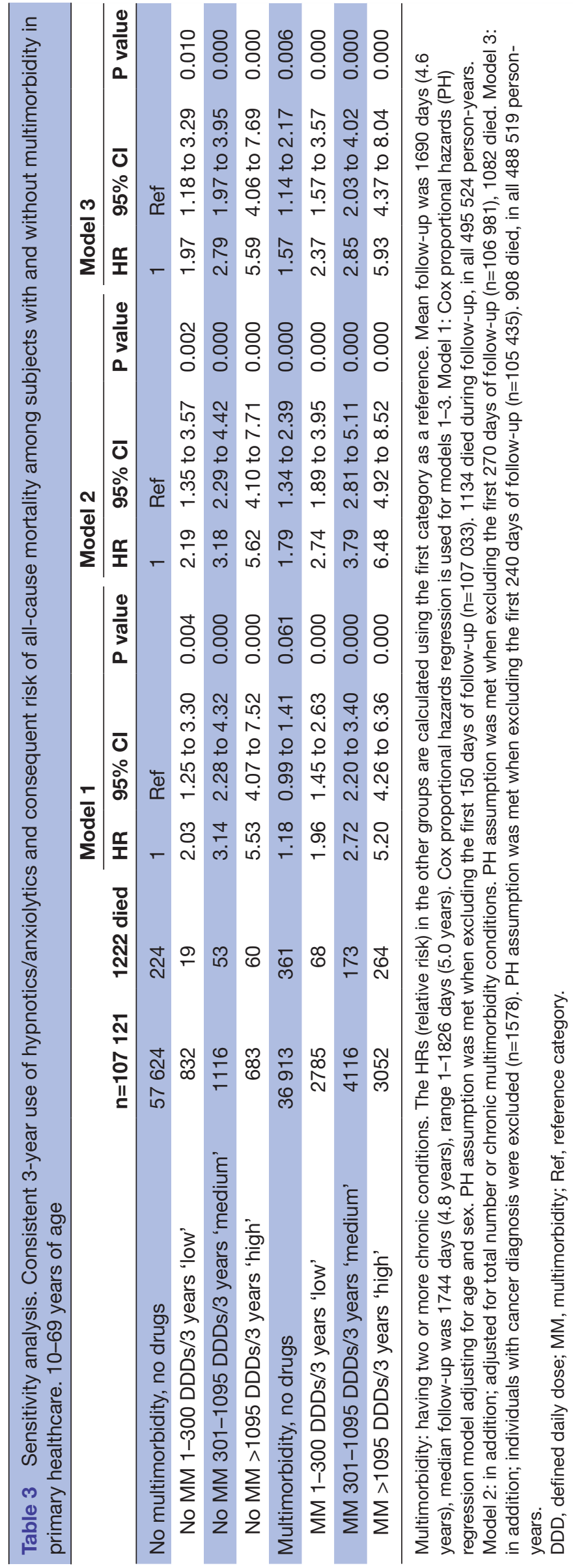



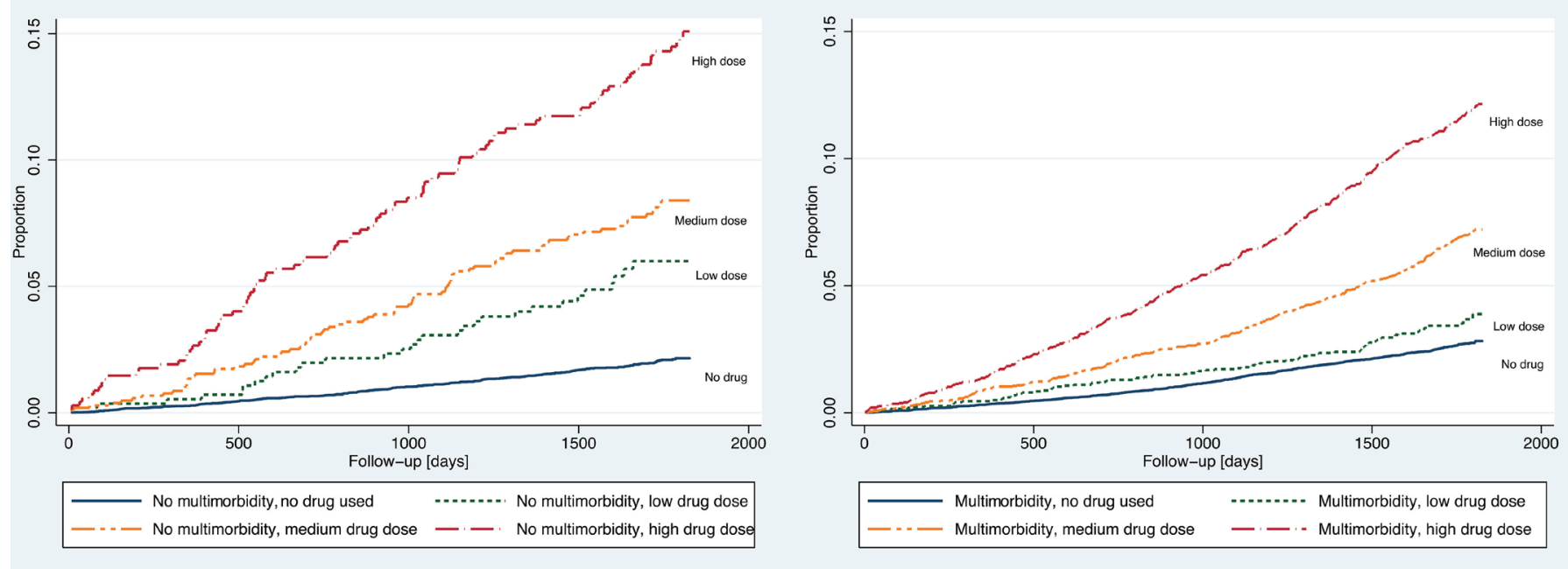

Figure 3 Dose-dependent mortality in relation to a 3-year consistent use of hypnotics/anxiolytics. Graph on the left: mortality (Nelson-Aalen cumulative hazard) among individuals aged 40-79 years, with no multimorbidity in relation to a 3-year consistent use of hypnotics/anxiolytics. Drug dose classified as: 'low' 1-300 defined daily doses (DDD) over 3 years before follow-up day 0; 'medium' 301-1095 DDDs over 3 years; 'high' >1095 DDDs/3 years, n=16 978. Patients with cancer diagnosis were excluded, $n=81$. Graph on the right: mortality (Nelson-Aalen cumulative hazard) among multimorbid patients aged 40-79 years, in relation to 3-year consistent use of hypnotics/anxiolytics in different doses. Drug dose classified as: 'low' 1-300 DDDs over 3 years before follow-up day 0; 'medium' 301-1095 DDDs/3 years; 'high' >1095 DDDs/3 years, n=32 420. Patients with cancer diagnosis were excluded, $\mathrm{n}=2089$ (Same classification of multimorbidity and drug use is also used in tables 1 and 2 ).

heterogeneous to a certain extent. The nature of the diseases, the disease combinations and the different number of diseases per patient above the minimum of 2 add to its variability. ${ }^{28}$ Furthermore, the burden of disease can vary depending on the severity and nature of the disease combinations, age of the patient, and so on, as well as its impact on the outcome, including mortality. A previous study revealed that in multimorbid, incident patients receiving hypnotic/anxiolytic prescriptions, a combination of mental disorders and pain-related diagnoses dominated the clinical profile. ${ }^{25}$ Potential confounders that we lack information on include use of other respiratory suppressant drugs, alcohol consumption, smoking, social status, education and income. Moreover, confounding by indication is inevitable. If we consider confounders not registered in the current study, residual confounding and potential non-adherence to treatment, then we may expect the HRs to attenuate to some degree.

\section{Multimorbidity}

In the aforementioned study, we explored the relationship between multimorbidity and the use of hypnotic and anxiolytic drugs. Multimorbid patients were far more likely to use hypnotics/anxiolytics compared with those without multimorbidity, OR=14.9 (95\% CI 14.4 to 15.4$).{ }^{25}$ Looking at the mortality of all the multimorbid patients who did not use hypnotics/anxiolytics, we found it to be around $14 \%$ higher $(95 \%$ CI $0 \%$ to $30 \%)$ than in patients neither multimorbid nor using hypnotic/anxiolytic drugs. This is comparable to increased mortality of multimorbid individuals reported in other studies. ${ }^{2324}$ Grouping participants by the number of chronic conditions, setting no multimorbidity (zero or one chronic condition) as a reference, revealed an increase in the risk of mortality among individuals with four to five conditions or more. The risk increased with increasing number of chronic conditions.

\section{Drug dose and morbidity}

We defined long-term use of hypnotics/anxiolytics relatively evenly spread over time as consistent use during a 3-year period. We divided the participants into low-dose, medium-dose and high-dose users. It may be argued whether the low-dose users can be called consistent users, as the intervals between prescriptions may at times have been somewhat irregular, but they redeemed the drugs every now and then during the 3 consecutive years being studied. Moreover, $75 \%$ of low-dose users took between 100 and 300 DDDs of the drugs during the 3-year recruitment period. Compared with mortality in patients neither multimorbid nor using hypnotics/anxiolytics, the cumulative hazard curves rise steadily with increasing dose for both multimorbid and non-multimorbid patients using hypnotics/ anxiolytics. To account for considerable differences in the average age between those who used hypnotics/anxiolytics and those who did not, the hazard curves were restricted to persons from 40 to 79 years of age. These results show that the contribution of multimorbidity to the mortality is fairly constant following the 3-year recruitment/exposure period under investigation, the increase in mortality first and foremost associated with the dose of hypnotics/ anxiolytics which might point to these drugs as a culprit in this increase in mortality. In the sensitivity analysis where persons aged 70-79 years were omitted, the risk of all-cause mortality in relation to drug dose increased consistently across all groups, from an adjusted HR 3.35 (persons 10-79 years of age) to HR 5.59 (persons aged 10-69 years) in 
non-multimorbid individuals, and from HR 3.52 to HR 5.93 in those who were multimorbid. This reflects a lower baseline risk in younger individuals and may have to be considered when prescribing these drugs.

In the second sensitivity analysis, when excluding the sexagenarians (60-69) the endpoints were too few in number, both in the low-dose and the medium-dose groups, for calculating HR. When omitting patients with cancer, the adjusted HR was 8.96 (95\% CI 5.72 to 14.02) for those with no multimorbidity and high dose of hypnotics/ anxiolytics, and similarly for those with multimorbidity and high dose of hypnotics/anxiolytics the adjusted HR was 11.92 (95\% CI 7.33 to 19.37 ). The risk observed in individuals aged 10-59 years using high doses of hypnotics/ anxiolytics is a further indication of higher risk of early mortality among younger persons associated with high-dose use of these drugs compared with corresponding groups of older persons. The third sensitivity analysis was done in order to resemble a cumulative dose for 6 weeks' treatment with hypnotics and/or anxiolytics. We cannot draw any conclusions regarding persons without multimorbidity, since only one endpoint occurred during the follow-up, but among those with multimorbidity the HR was 1.13 (95\% CI 0.53 to 2.40) which does not indicate an increased risk of mortality for using up to 42 DDDs/year of hypnotics and/ or anxiolytics. A Danish study involving men born in 1953 living in Denmark 1 January $1996^{29}$ did not find an association between mortality and low-dose use of hypnotics (an average of $\leq 30$ DDDs/year, HR=1.22, 95\% CI 0.97 to 1.54 ). An association was found in high-dose users (ie, $>30$ DDDs/ year, $\mathrm{HR}=1.43,95 \%$ CI 1.11 to 1.85 ). This is in accordance with our results. In the above-mentioned sensitivity analysis multimorbid patients using 42 DDDs/year were not at increased risk of mortality.

In a retrospective, matched cohort study on the effect of anxiolytic and hypnotic prescriptions on mortality hazards, ${ }^{9}$ the risk followed a dose-response pattern both for benzodiazepines and Z-drugs and did not seem to be entirely due to confounding by physical or psychiatric comorbidity or prescribing of other drugs. In that study an exposure of 1-30 DDDs of hypnotic/anxiolytic drugs was associated with HR 2.55 (95\% CI 2.42 to 2.69), and an exposure of $\geq 91$ DDDs was associated with HR 4.51 (95\% CI 4.22 to 4.82). When the analysis was confined to subjects with a 12-month follow-up or more, the corresponding HRs were attenuated, 1.45 (95\% CI 1.35 to 1.56 ) and 2.63 (95\% CI 2.34 to 2.95 ), thus indicating a dose-response, which is in accordance with our findings.

\section{Different interpretations of results}

Other views have been presented, for example, in a study connecting disease burden and use of hypnotics, showing a $50 \%$ increase in the use of benzodiazepines 24 months before death. The authors concluded that the association between use of these drugs and mortality is at least partially due to confounding. ${ }^{30}$ A Finnish study where the purchase of hypnotic drugs registered in a nationwide database was followed for up to 7.6 years, deaths in the cohort were matched with control cases. According to the authors' interpretation the developmental trajectories of purchases were differently and plausibly associated with specific mortality risks. They concluded that the association was created without specific biological mechanisms by symptomatic treatment of discomfort caused by terminal illness. ${ }^{31}$ In our study, we have eliminated persons closely approaching death by requiring a 3-year period of consistent use, and subsequently, for the participants for whom follow-up for mortality was started, the average follow-up time was 4.6 years. In addition, to address the potential confounding effects of terminal illness, subjects with cancer diagnosis were excluded in risk estimates (table 2, model 3; table 3, model 3). These measures reduce the risk of reverse causation bias.

\section{Influence of pharmacological effects}

The pharmacological properties of hypnotics could play a role in mortality in these patients as mentioned in the Introduction section. The respiratory suppressant effects of benzodiazepines and related drugs increase in combination with other respiratory depressant drugs or alcohol. ${ }^{10}$ In the Introduction section, we already mentioned coadministration of opioids and benzodiazepines, ${ }^{11} 12$ and also use of psychotropic drugs leading to increased risk of falls and fractures and as a consequence increased mortality in the elderly, ${ }^{13-15}$ the possible association with increased suicide risk, ${ }^{316}$ increased risk of road traffic accidents, ${ }^{17}$ dementia ${ }^{18}$ and Alzheimer's disease. ${ }^{1920}$ Exposure to benzodiazepines has been found to be associated with an increased risk of community-acquired pneumonia and consequently increased mortality. ${ }^{22} \mathrm{~A}$ Canadian population-based cohort study found that when adults aged 66 years and older with chronic obstructive pulmonary disease were examined within 30 days of incident benzodiazepine use, new benzodiazepine users were at significantly higher risk for outpatient respiratory exacerbation compared with non-users. ${ }^{32}$ Evidently, the above-mentioned points could all contribute to increased mortality, separately and/or jointly. Multimorbidity as such is associated with increased mortality. ${ }^{2324}$

\section{CONCLUSIONS}

The increase in mortality with increasing doses of hypnotics/ anxiolytics in both the multimorbid patients and those without multimorbidity might possibly be a consequence of the use of these drugs as there is a dose-dependent increase in mortality in both categories of patients with a similar difference between the corresponding groups (multimorbid - non-multimorbid). Our findings support the general recommendations that the use of benzodiazepines and Z-drugs should be limited to a period of 2-4 up to 6 weeks, long-term use being associated with increased risk. When possible, non-pharmacological approaches should be sought to treat long-term sleeping problems, for example, cognitive-behavioural therapy and by teaching better sleeping habits. ${ }^{33-35}$ 


\section{Author affiliations}

${ }^{1}$ Development Centre for Primary Healthcare in Iceland, Primary Health Care of the Capital Area, Reykjavik, Iceland

${ }^{2}$ General Practice Research Unit, Department of Public Health and Nursing, Norwegian University of Science and Technology (NTNU), Trondheim, Norway ${ }^{3}$ Department of Family Medicine, Faculty of Medicine, University of Iceland School of Health Sciences, Reykjavik, Iceland

${ }^{4}$ Faculty of Pharmaceutical Sciences, School of Health Sciences, University of Iceland, Reykjavik, Iceland

Contributors KL, LSG and JAS initiated and designed the study. KL, LSG, JAS, MOT and ELS performed further development. KL and LSG drafted the manuscript with input from JAS which was critically reviewed by all authors. LSG performed the statistical analysis. KL, LSG, JAS, MOT and ELS read and approved the final version of the manuscript.

Funding This research was supported by the Research Fund of the Icelandic College of Family Physicians and the Fund of Scientific Research of the Pharmaceutical Society of Iceland.

Competing interests None declared.

Patient consent for publication Not required.

Provenance and peer review Not commissioned; externally peer reviewed.

Data availability statement The data is retrieved from a medical records database in the Primary Healthcare of the Capital Area (PHCA), the Icelandic Medicine Register (IMR) and the National Cause of Death Registry of the Directorate of Health. The encrypted data is kept at the Directorate of Health and can made available on a reasonable request if permitted by the above-mentioned health authorities.

Open access This is an open access article distributed in accordance with the Creative Commons Attribution Non Commercial (CC BY-NC 4.0) license, which permits others to distribute, remix, adapt, build upon this work non-commercially, and license their derivative works on different terms, provided the original work is properly cited, appropriate credit is given, any changes made indicated, and the use is non-commercial. See: http://creativecommons.org/licenses/by-nc/4.0/.

\section{ORCID iD}

Kristjan Linnet http://orcid.org/0000-0002-0189-9519

\section{REFERENCES}

1 Kripke DF, Langer RD, Kline LE. Hypnotics' association with mortality or cancer: a matched cohort study. BMJ Open 2012;2:e000850.

2 Belleville G. Mortality hazard associated with anxiolytic and hypnotic drug use in the National population health survey. Can J Psychiatry 2010;55:558-67.

3 Mallon L, Broman J-E, Hetta J. Is usage of hypnotics associated with mortality? Sleep Med 2009;10:279-86.

4 Winkelmayer WC, Mehta J, Wang PS. Benzodiazepine use and mortality of incident dialysis patients in the United States. Kidney Int 2007;72:1388-93.

5 Hartz A, Ross JJ. Cohort study of the association of hypnotic use with mortality in postmenopausal women. BMJ Open 2012;2:e001413.

6 Gisev N, Hartikainen S, Chen TF, et al. Mortality associated with benzodiazepines and benzodiazepine-related drugs among community-dwelling older people in Finland: a population-based retrospective cohort study. Can J Psychiatry 2011;56:377-81.

7 Patorno E, Glynn RJ, Levin R, et al. Benzodiazepines and risk of all cause mortality in adults: cohort study. BMJ 2017;358:j2941.

8 Jaussent I, Ancelin M-L, Berr C, et al. Hypnotics and mortality in an elderly general population: a 12-year prospective study. BMC Med 2013;11:212.

9 Weich S, Pearce HL, Croft P, et al. Effect of anxiolytic and hypnotic drug prescriptions on mortality hazards: retrospective cohort study. BMJ 2014;348:g1996.

10 Gutstein HB, Akil H. Opioid analgesics. In: Brunton LL, Lazo JS, Parker KL, eds. Goodman and Gilman's the pharmacological basis of therapeutics. 560. 11th edn. New York: McGraw-Hill, 2006.
11 Park TW, Saitz R, Ganoczy D, et al. Benzodiazepine prescribing patterns and deaths from drug overdose among US veterans receiving opioid analgesics: case-cohort study. BMJ 2015;350:h2698.

12 Sigurdsson MI, Helgadottir S, Long TE, et al. Association between preoperative opioid and benzodiazepine prescription patterns and mortality after noncardiac surgery. JAMA Surg 2019;154:e191652.

13 Thorell K, Ranstad K, Midlöv P, et al. Is use of fall risk-increasing drugs in an elderly population associated with an increased risk of hip fracture, after adjustment for multimorbidity level: a cohort study. BMC Geriatr 2014;14:131.

14 Cumming RG, Le Couteur DG. Benzodiazepines and risk of hip fractures in older people: a review of the evidence. CNS Drugs 2003;17:825-37.

15 Katelaris AG, Cumming RG. Health status before and mortality after hip fracture. Am J Public Health 1996;86:557-60.

16 Carlsten A, Waern M. Are sedatives and hypnotics associated with increased suicide risk of suicide in the elderly? BMC Geriatr 2009;9:20.

17 Gustavsen I, Bramness JG, Skurtveit S, et al. Road traffic accident risk related to prescriptions of the hypnotics zopiclone, zolpidem, flunitrazepam and nitrazepam. Sleep Med 2008;9:818-22.

18 Billioti de Gage S, Bégaud B, Bazin F, et al. Benzodiazepine use and risk of dementia: prospective population based study. $B M J$ 2012;345:e6231.

19 Billioti de Gage S, Moride Y, Ducruet T, et al. Benzodiazepine use and risk of Alzheimer's disease: case-control study. BMJ 2014;349:g5205.

20 Saarelainen L, Tolppanen A-M, Koponen M, et al. Risk of death associated with new benzodiazepine use among persons with Alzheimer disease: a matched cohort study. Int J Geriatr Psychiatry 2018;33:583-90.

21 Gray SL, Dublin S, Yu O, et al. Benzodiazepine use and risk of incident dementia or cognitive decline: prospective population based study. BMJ 2016;352.

22 Obiora E, Hubbard R, Sanders RD, et al. The impact of benzodiazepines on occurrence of pneumonia and mortality from pneumonia: a nested case-control and survival analysis in a population-based cohort. Thorax 2013;68:163-70.

23 Gijsen R, Hoeymans N, Schellevis FG, et al. Causes and consequences of comorbidity: a review. J Clin Epidemiol 2001:54:661-74.

24 Loprinzi PD, Addoh O, Joyner C. Multimorbidity, mortality, and physical activity. Chronic IIIn 2016;12:272-80.

25 Linnet K, Gudmundsson LS, Birgisdottir FG, et al. Multimorbidity and use of hypnotic and anxiolytic drugs: cross-sectional and follow-up study in primary healthcare in Iceland. BMC Fam Pract 2016;17:69.

26 Grambsch PM, Therneau TM. Proportional hazards tests and diagnostics based on weighted residuals. Biometrika 1994;81:515-26.

27 Schoenfeld D. Partial residuals for the proportional hazards regression model. Biometrika 1982;69:239-41.

28 Ramond-Roquin A, Haggerty J, Lambert M, et al. Different multimorbidity measures result in varying estimated levels of physical quality of life in individuals with multimorbidity: a cross-sectional study in the general population. Biomed Res Int 2016;2016:7845438.

29 Neutel $\mathrm{Cl}$, Johansen $\mathrm{HL}$. Association between hypnotics use and increased mortality: causation or confounding? Eur J Clin Pharmacol 2015;71:637-42.

30 Kriegbaum M, Hendriksen C, Vass M, et al. Hypnotics and mortality partial confounding by disease, substance abuse and socioeconomic factors? Pharmacoepidemiol Drug Saf 2015;24:779-83.

31 Kronholm E, Jousilahti P, Laatikainen T, et al. Trajectories in hypnotic use and approaching death: a register linked case-control study. Sleep Med 2019;57:153-61.

32 Vozoris NT, Fischer HD, Wang X, et al. Benzodiazepine drug use and adverse respiratory outcomes among older adults with COPD. Eur Respir J 2014;44:332-40.

33 Jacobs GD, Pace-Schott EF, Stickgold R, et al. Cognitive behavior therapy and pharmacotherapy for insomnia: a randomized controlled trial and direct comparison. Arch Intern Med 2004;164:1888-96.

34 Espie CA. 'Stepped care': a health technology solution for delivering cognitive behavioral therapy as a first line insomnia treatment. Sleep 2009:32:1549-58.

35 Mitchell MD, Gehrman P, Perlis M, et al. Comparative effectiveness of cognitive behavioral therapy for insomnia: a systematic review. BMC Fam Pract 2012;13:40. 